\title{
GRADED RINGS OF COHOMOLOGICAL DIMENSION 2
}

\author{
Q. S. WU \\ Institute of Mathematics, Fudan University, Shanghai, 200433, China \\ e-mail:cwu@math.washington.edu,qswu@fudan.edu.cn \\ and J. J. ZHANG \\ Department of Mathematics, Box 354350, University of Washington, Seattle, WA 98195, USA \\ e-mail:zhang@math.washington.edu
}

(Received 18 January, 1999)

\begin{abstract}
Let $A$ be a noetherian connected graded ring with a balanced dualizing complex $R$. If $A$ has cohomological dimension and Krull dimension 2, then

(1) $R$ is Auslander;

(2) $\operatorname{Cdim} M=\mathrm{K} \operatorname{dim} M$ for all noetherian graded $A$-modules $M$.

In particular, if $A$ is AS-Gorenstein of injective and Krull dimension 2, then

(3) $A$ is Auslander-Gorenstein;

(4) $A$ is 2-pure with a self-injective artinian quotient ring;

(5) $A$ has a residue complex.

$(1,3,4)$ generalize a result of Levasseur $[7,5.13]$ and (5) generalizes a result of Ajitabh [1, 3.12].
\end{abstract}

1991 Mathematics Subject Classification. 16E10, 16W50.

0. Introduction. Auslander property is closely related to other properties such as catenarity, localizability, and existence of nice dimension function (see [16]). The Auslander property is proved for some classes of rings such as AS-regular rings of global dimension no more than $3[\mathbf{2}, \mathbf{3}, \mathbf{7}]$ and the Sklyanin algebras $[\mathbf{1 1}, \mathbf{1 2}]$. It is an open question whether or not every noetherian AS-Gorenstein ring is AuslanderGorenstein. Levasseur $[7,5.13]$ proved that a noetherian AS-Gorenstein ring $A$ of injective dimension 2 is Auslander-Gorenstein if $A$ has Gelfand-Kirillov dimension 2 and $A$ is 2-homogeneous (i.e., 2-pure) with a self-injective artinian quotient ring. The main object of this note is to generalize Levasseur's result. The following is proved in Section 3.

THEOREM 0.1. Let $A$ be a noetherian connected graded ring with a balanced dualizing complex $R$. Suppose A has cohomological dimension 2. Then the following are equivalent:

(1) $R$ is Auslander.

(2) $\operatorname{Kdim} A=2$ where Kdim denotes the Krull dimension.

(3) there is a graded dimension function $\partial$ such that $\partial A<3$.

(4) for any chain of graded primes $P^{\prime} \subsetneq P \subset A$, $\operatorname{Kdim} A / P \leq 1$.

(5) the canonical dimension $\mathrm{Cdim}$ is a graded dimension function.

(6) Cdim is exact.

(7) $\mathrm{Cdim} M=\mathrm{K} \operatorname{dim} M$ for all noetherian graded A-modules $M$.

The definitions of the Auslander property and Gorenstein property are given next. 
Definition 0.2. A balanced dualizing complex $R$ over $A$ is Auslander if for every noetherian graded $A$-module $M$ and for every graded $A$-submodule $N \subset \operatorname{Ext}^{i}(M, R), \operatorname{Ext}^{j}(N, R)=0$ for all $j<i$.

Definition 0.3. (1) A noetherian connected graded ring $A$ is called $A S$-Gorenstein (where AS stands for Artin and Schelter) if $A$ has injective dimension $d<\infty$ and $\operatorname{Ext}^{i}(k, A)=0$ for $i \neq d$ and $\operatorname{Ext}^{d}(k, A)=k(e)$ for some $e \in \mathbb{Z}$, where $k$ is viewed as either left or right trivial $A$-module.

(2) A noetherian (graded) ring $A$ is called (graded) Auslander-Gorenstein if $A$ has finite injective dimension and for every noetherian (graded) $A$-module $M$ and for every (graded) $A$-submodule $N \subset \operatorname{Ext}^{i}(M, A), \operatorname{Ext}^{j}(N, A)=0$ for all $j<i$.

We refer to $[13,14,15,16]$ for other definitions and notations.

By [7, 6.3] every connected graded Auslander-Gorenstein ring is AS-Gorenstein. By [5, 0.1], a noetherian graded ring is Auslander-Gorenstein if and only if it is graded Auslander-Gorenstein. By [14, 4.14], AS-Gorenstein rings have balanced dualizing complexes and in this case the injective dimension of $A$ is its cohomological dimension. By [15, Section 0], a connected graded ring is Auslander-Gorenstein if and only if it is AS-Gorenstein and its balanced dualizing complex is Auslander.

In addition to Theorem 0.1, we prove the following for AS-Gorenstein rings in Section 4.

Theorem 0.4. Let A be a noetherian AS-Gorenstein ring of injective dimension 2. Then the following are equivalent (and equivalent to the conditions in Theorem 0.1):

(1) A is Auslander-Gorenstein.

(2) A has a self-injective artinian quotient ring.

(3) A has an artinian quotient ring.

(4) for every minimal prime ideal $P \subset A, \operatorname{Kdim} A / P=2$.

(5) A has a residue complex.

(6) $A$ is 2-pure, i.e., for every nonzero left (right) ideal $I \subset A, \operatorname{Kdim} I=2$.

We are unable to show that every noetherian connected graded ring of cohomological dimension 2 has Krull dimension 2. The following partial result is proved in Section 2.

Proposition 0.5. Let $A$ be a noetherian connected graded ring with a balanced dualizing complex. If $A$ has cohomological dimension 2, then

(1) $\mathrm{Kdim}_{A^{e}} A \leq 2$, where $A^{e}=A \otimes A^{o p}$,

(2) there are only finitely many graded prime ideals $P$ with $\operatorname{Kdim} A / P \geq 2$.

1. Balanced dualizing complexes. Let $k$ be a base field and $A$ a connected graded ring over $k$, i.e., $A=k \oplus A_{1} \oplus A_{2} \oplus \cdots$. Unless otherwise stated we are working on connected graded rings over $k$ and their graded modules. If $A$ is left (or right) noetherian, then each $A_{i}$ is finite dimensional over $k$. Let $\mathfrak{m}$ denote the maximal graded ideal $A_{\geq 1}$ of $A$. The $\mathfrak{m}$-torsion submodule of $M$, denoted by $\Gamma_{\mathfrak{m}}(M)$, is the union of all finite dimensional submodules of $M$. The left (right) cohomological dimension of $A$ is defined to be the cohomological dimension of $\Gamma_{\mathfrak{m}}$ applying to the left (right) graded $A$-modules. 
Our main tool is the balanced dualizing complex introduced by Yekutieli [14]. A theorem of Van den Bergh [13, 6.3] states as follows: A noetherian connected graded ring $A$ has a balanced dualizing complex if and only if $A$ satisfies left and right $\chi$ condition and has finite left and right cohomological dimension. Both $\chi$-condition and having finite cohomological dimension are checked for a large class of noetherian graded rings $[\mathbf{4}, \mathbf{1 6}]$.

From now on $A$ is a noetherian connected graded ring with a balanced dualizing complex $R$. It follows from $[\mathbf{1 3}, 4.8]$ that the left cohomological dimension of $A$ is equal to the right cohomological dimension of $A$, which is denoted by $\operatorname{cd} A$.

Let $M$ be a graded $A$-module. The graded vector space dual of $M$ is $M^{\prime}=\oplus_{n} \operatorname{Hom}_{k}\left(M_{-n}, k\right)$. The local duality theorem states the following: for any $X \in D(\mathrm{Gr} A)$,

$$
\mathrm{R} \Gamma_{\mathfrak{m}}(X)^{\prime}=\mathrm{RHom}_{A}(X, R)
$$

(see $[14,4.18]$ and $[13,5.1])$.

A dualizing complex $R$ induces a convergent spectral sequence: for any noetherian module $M$

$$
E_{2}^{p, q}:=\operatorname{Ext}^{p}\left(\operatorname{Ext}^{q}(M, R), R\right) \Longrightarrow \mathbb{H}^{p-q}(M)
$$

where $\mathbb{H}^{p-q}(M)=0$ if $p \neq q$ and $\mathbb{H}^{0}(M)=M[\mathbf{1 6}, 1.7]$.

Suppose $A$ has cohomological dimension $d$ with a balanced dualizing complex $R$. It follows from the local duality theorem (E1.1) that, for all $i>0$ and $i<-d$,

$$
\operatorname{Ext}^{i}(M, R)=0
$$

for all left (or right) $A$-modules $A$. Hence we may choose $R$ to be a complex in $D_{f g}^{b}\left(\mathrm{Gr} A^{e}\right)$ of the form

$$
R=\cdots 0 \rightarrow R^{-d} \rightarrow \cdots \rightarrow R^{-1} \rightarrow R^{0} \rightarrow 0 \cdots
$$

where each $R^{q}$ is injective as left and as right $A$-module.

Lemma 1.3. Let $M$ be a noetherian graded A-module.

(1) $\operatorname{Ext}^{0}(M, R)=\Gamma_{\mathfrak{m}}(M)^{\prime}$.

(2) If $\operatorname{dim}_{k} M<\infty$, then $\operatorname{Ext}^{q}(M, R)=0$ for all $q \neq 0$ and $\operatorname{Ext}^{0}(M, R)=M^{\prime}$.

(3) If $\operatorname{dim}_{k} M=\infty$, then $\operatorname{dim}_{k} \operatorname{Ext}^{q}(M, R)=\infty$ for some $q<0$.

Proof. $(1,2)$ Follow from the local duality (E1.1).

(3) Suppose that $\operatorname{Ext}^{q}(M, R)$ is finite dimensional for all $q<0$. By (1) $\operatorname{Ext}^{0}(M, R)$ is finite dimensional. Hence, by (2), $\operatorname{Ext}^{p}\left(\operatorname{Ext}^{q}(M, R), R\right)$ is finite dimensional for all $p, q$. It follows from the spectral sequence (E1.2) that $M$ is finite dimensional.

If $\operatorname{cd} A=0$, then the short exact sequence $0 \longrightarrow \mathfrak{m} \longrightarrow A \longrightarrow A / \mathfrak{m} \longrightarrow 0$ induces an exact sequence

$$
0 \longrightarrow \Gamma_{\mathfrak{m}}(\mathfrak{m}) \longrightarrow \Gamma_{\mathfrak{m}}(A) \longrightarrow A / \mathfrak{m} \longrightarrow \mathrm{R}^{1} \Gamma_{\mathfrak{m}}(\mathfrak{m})=0 .
$$


Hence $1 \in \Gamma_{\mathfrak{m}}(A)$, so $A=\Gamma_{\mathfrak{m}}(A)$ is finite dimensional. Next we consider the case $\operatorname{cd} A=1$. Let GKdim denote the Gelfand-Kirillov dimension.

Lemma 1.4. Let $A$ and $B$ be noetherian graded rings.

(1) If $\mathrm{GK} \operatorname{dim} A=1$, then $A$ is $P I$ (PI=satisfying a polynomial identity) and $\operatorname{Kdim} A=1$.

(2) If $\mathrm{Kdim} A=1$, then $\mathrm{GKdim} A=1$.

(3) If $M$ is a graded $(A, B)$-module noetherian on both sides, then the following are equivalent:

(a) $\mathrm{Kdim} M_{B}=1$, (b) $\mathrm{GKdim} M_{B}=1$, (c) $1 \leq \mathrm{GKdim} M_{B}<2$, (d) $\mathrm{GKdim}_{A} M=1$.

Proof. (1) By [10], if $\mathrm{GKdim} A=1$, then $A$ is PI. By [8, 6.4.8 and 13.10.6] $\mathrm{K} \operatorname{dim} A=\mathrm{GK} \operatorname{dim} A=1$.

(2) We may assume $A$ is prime. By a graded version of Goldie's theorem [9, I.1.6], there is a homogeneous regular element $x \in A$ of degree $d>0$. Hence $\mathrm{K} \operatorname{dim} A / x A=0$, which implies that $A / x A$ is finite $\operatorname{dimensional.~Hence~} \mathrm{GK} \operatorname{dim} A=1$.

(3) By noetherian induction we may assume $M$ is critical and $\operatorname{ann}\left({ }_{A} M\right)=: Q$ and $\operatorname{ann}\left(M_{B}\right)=: P$ are graded prime ideals such that ${ }_{A} M$ is $A / Q$-torsionfree and $M_{B}$ is $B / P$-torsionfree. Hence $\mathrm{K} \operatorname{dim} M_{B}=\mathrm{K} \operatorname{dim} B / P$ and $\mathrm{GKdim} M_{B}=\mathrm{GKdim} B / P$. Now (a) and (b) are equivalent by $(1,2)$, and (b) and (c) are equivalent by Bergman's gap theorem $[6,2.5]$. (b) and (d) are equivalent because GKdim is symmetric $[\mathbf{6}, 5.4]$.

Lemma 1.5. If $\operatorname{cd} A=1$, then $A$ is PI of $\mathrm{Kdim} 1$. More generally, if $M$ is a noetherian graded $A$-module such that (a) $\operatorname{Ext}^{-i}(M, R)=0$ for all $i>1$ and (b) $f: M(-d) \rightarrow M$ is an injective map for some $d>0$, then $M / f(M)$ is finite dimensional.

Proof. We may assume that $A$ is prime $[4,8.5]$ and not $k$. By $[9$, I.1.6], there is a homogeneous regular element $x \in A$ of degree $d>0$. Then there is a short exact sequence

$$
0 \rightarrow A(-d) \rightarrow A \rightarrow A / x A \rightarrow 0
$$

By Lemma 1.4 , it suffices to show $\operatorname{GKdim} A=1$. To prove $\operatorname{GKdim} A=1$, it suffices to prove that $A / x A$ is finite dimensional over $k$.

We now consider the general case when $0 \rightarrow M(-d) \rightarrow M \rightarrow M / f(M) \rightarrow 0$ is exact and show that $M / f(M)$ is finite dimensional. By the long exact sequence we see that

$$
\begin{gathered}
\operatorname{Ext}^{-i}(M / f(M), R)=0 \text { for all } i>1, \quad \text { and } \\
0 \rightarrow \operatorname{Ext}^{-1}(M / f(M), R) \rightarrow \operatorname{Ext}^{-1}(M, R) \rightarrow \operatorname{Ext}^{-1}(M(-d), R) \rightarrow \operatorname{Ext}^{0}(M / f(M), R)
\end{gathered}
$$

is exact. Let $f^{n}(M)$ be the image of the map $f^{n}: M(-d n) \rightarrow M$. Then $f^{n}(M) / f^{n+1}(M) \cong(M / f(M))(-d n)$. The short exact sequence

$$
0 \rightarrow f^{n}(M) / f^{n+1}(M) \rightarrow M / f^{n+1}(M) \rightarrow M / f^{n}(M) \rightarrow 0
$$

yields a long exact sequence 


$$
\begin{aligned}
0 \rightarrow \operatorname{Ext}^{-1}\left(M / f^{n}(M), R\right) \rightarrow & \operatorname{Ext}^{-1}\left(M / f^{n+1}(M), R\right) \rightarrow \\
& \operatorname{Ext}^{-1}\left(f^{n}(M) / f^{n+1}(M), R\right) \rightarrow \operatorname{Ext}^{0}\left(M / f^{n}(M), R\right) .
\end{aligned}
$$

Suppose $M / f(M)$ is infinite dimensional. By Lemma 1.3(3), $\operatorname{Ext}^{-1}(M / f(M), R)$, and hence $\operatorname{Ext}^{-1}\left(f^{n}(M) / f^{n+1}(M), R\right)$, is infinite dimensional. Since $\operatorname{Ext}^{0}\left(M / f^{n}(M), R\right)$ is finite dimensional, the injective map

$$
\operatorname{Ext}^{-1}\left(M / f^{n}(M), R\right) \rightarrow \operatorname{Ext}^{-1}\left(M / f^{n+1}(M), R\right)
$$

is not surjective. This shows that $\left\{\operatorname{Ext}^{-1}\left(M / f^{n}(M), R\right) \mid n \geq 0\right\}$ is an ascending chain of submodules of $\operatorname{Ext}^{-1}(M, R)$, which contradicts to the fact that $\operatorname{Ext}^{-1}(M, R)$ is noetherian.

Let $H^{i} R$ be the $i$-th cohomology of $R$. By definition, $H^{i} R$ is noetherian on both sides for all $i$.

Lemma 1.6. Suppose $\operatorname{cd} A=d \geq 2$ and $M$ is a noetherian graded A-module.

(1) $H^{-d} R$ is noetherian on both sides and $\operatorname{Ext}^{-d}(M, R)=\operatorname{Hom}\left(M, H^{-d} R\right)$.

(2) Every nonzero left (right) submodule of $H^{-d} R$ has $\mathrm{Kdim} \geq 2$.

(3) If $A$ is prime such that, for every nonzero graded prime $P, \mathrm{Kdim} A / P \leq 1$, then $H^{-d} R$ is $A$-torsionfree on both sides. Namely, $\operatorname{Ext}^{-d}(M, R)=0$ for all $A$ torsion modules $M$.

Proof. (1) Clear.

(2) For any $\alpha$, the maximal submodule of $H^{-d} R$ of Krull dimension at most $\alpha$ is a graded submodule. Hence it suffices to consider graded submodules. Suppose $N$ is a right graded submodule of $H^{-d} R$ of $\mathrm{Kdim}<2$. Since $H^{-d} R$ is noetherian on both sides, we may further assume that (a) $N$ is a critical subbimodule of $H^{-d} R$, (b) $P:=\operatorname{ann}\left(N_{A}\right)$ is prime, and (c) $N$ is $A / P$-torsionfree $[8,4.3 .5($ iii) $]$. Hence $\mathrm{K} \operatorname{dim} A / P=\mathrm{K} \operatorname{dim} N_{A}<2$, and then $\mathrm{Kdim} A / P \leq 1$. By (1) and Lemma 1.3(2), $H^{-d} R$ contains no finite dimensional submodule. Thus $\mathrm{Kdim} A / P=1$. By Lemma 1.4, $A / P$ is PI, whence there is a central regular elements $x \in A / P$ of degree $d>0$. The short exact sequence

$$
0 \rightarrow N(-d) \rightarrow N \rightarrow N / N x \rightarrow 0
$$

yields a long exact sequence

$$
\operatorname{Ext}^{-d}(N / N x, R) \rightarrow \operatorname{Ext}^{-d}(N, R) \rightarrow \mathrm{Ext}^{-d}(N(-d), R) \rightarrow \operatorname{Ext}^{-d+1}(N / N x, R) .
$$

Since $N / N x$ is finite dimensional, two ends are zero [Lemma 1.3(2)]. Since $\operatorname{Ext}^{-d}(N, R)$ is left bounded, a graded version of Nakayama lemma implies that $\operatorname{Ext}^{-d}(N, R)$ is zero. This contradicts to the fact $N \subset H^{-d} R$.

(3) Suppose $H^{-d} R$ is not $A$-torsionfree. Since $H^{-d} R$ is noetherian on both sides, there is a critical subbimodule $N \subset H^{-d} R$ such that (a) $N_{A}$ is $A$-torsion, (b) $P:=\operatorname{ann}\left(N_{A}\right)$ is a nonzero graded prime ideal, and (c) $N$ is $A / P$-torsionfree. By the hypothesis, $\mathrm{K} \operatorname{dim} N_{A}=\mathrm{K} \operatorname{dim} A / P \leq 1$. This contradicts to (2).

If $P$ is a graded prime ideal of $A$, let $E(A / P)$ denote the graded injective hull of $A / P$ and $E_{A / P}$ denote the graded injective hull of a graded uniform right ideal of $A / P$. 
Lemma 1.7. Let I be the minimal injective resolution of $R$ as right A-module complex.

(1) $I^{0}=A^{\prime}$.

(2) Let $B^{-i}$ be the image of $I^{-i-1} \rightarrow I^{-i}$. Then $I^{-1} / B^{-1}$ is locally finite.

(3) If $A$ is prime and $\operatorname{Kdim} A \geq 2$, then $I^{-1}$ does not contain any shift of the injective module $E_{A}$.

(4) If $P$ is a graded prime ideal of $A$ such that $\mathrm{Kdim} A / P \geq 2$, then $I^{-1}$ does not contain any shift of the injective module $E_{A / P}$.

Proof. (1) By local duality (E1.1), $\operatorname{Ext}^{0}(k, R)=\Gamma_{\mathfrak{m}}(k)^{\prime}=k$. Thus $I^{0}=A^{\prime} \oplus J$ where $J$ is $\mathfrak{m}$-torsionfree. Suppose $I^{0} \neq A^{\prime}$, namely, $J \neq 0$. Then the inclusion $J \rightarrow I^{0}$ induces a nonzero element in $\operatorname{Ext}^{0}(J, R)$. Since $J$ is $\mathfrak{m}$-torsionfree, by local duality $\operatorname{Ext}^{0}(J, R)=\Gamma_{\mathfrak{m}}(J)^{\prime}=0$, a contradiction.

(2) We have an exact sequence

$$
0 \rightarrow H^{-1} R \rightarrow I^{-1} / B^{-1} \rightarrow I^{0} .
$$

By (1) $I^{0}=A^{\prime}$ is locally finite. By the definition of dualizing complex, $H^{-1} R$ is noetherian and hence locally finite. The statement follows.

(3) Let $Q_{g r}(A)$ be the graded quotient ring of $A$. It is clear that $E(A)$ is isomorphic to $Q_{g r}(A)$ as left and as right graded $A$-module. If $E(A)$ is locally finite, then the Hilbert series of $E(A)=Q_{g_{r}}(A)$ is periodic. Hence $A$ has GKdim 1, which contradicts to the hypothesis. Therefore $E(A)$ is not locally finite. Since $E(A)$ is a finite direct sum of shifts of $E_{A}, E_{A}$ is not locally finite.

Suppose $E_{A}$ is a submodule of $I^{-1}$. Since $A$ is prime and $B^{-1}$ is $A$-torsion, $E_{A}$ does not intersect with $B^{-1}$. Hence $E_{A}$ is a submodule of $I^{-1} / B^{-1}$. By (2), $E_{A}$ is locally finite, a contradiction.

(4) Let $R_{A / P}$ be the dualizing complex over $A / P$. By [16, 3.2 and 4.16], there is a morphism $\phi: R_{A / P} \rightarrow R$ in $D\left(\operatorname{Gr} A^{e}\right)$ such that

$$
R_{A / P} \cong \mathrm{RHom}_{A}(A / P, R) \cong \mathrm{RHom}_{A^{\circ}}(A / P, R) .
$$

Note that if $M$ is an injective $A$-module, then $\operatorname{Hom}(A / P, M)$ is an injective $A / P$ module. Hence $I_{A / P}:=\operatorname{Hom}(A / P, I)$ is a complex of injective $A / P$-modules. The minimality of the complex $I$ implies that $I_{A / P}$ is a minimal complex of injective $A / P$ modules. By (E1.8), $I_{A / P}$ is a minimal injective resolution of $R_{A / P}$. Suppose $I^{-1}$ contains injective $A$-module $E_{A / P}$. Then $I_{A / P}^{-1}$ contains injective $A / P$-module $E_{A / P}$, which contradicts with (3).

2. Rings with cohomological dimension 2. By Lemma 1.5 , if $\operatorname{cd} A=1$ then $A$ is $\mathrm{PI}$ of $\mathrm{Kdim} 1$, which implies that $R$ is Auslander. In this section we will discuss the case $\operatorname{cd} A=2$. If $\operatorname{Kdim} A=1$, then $A$ is PI [Lemma 1.4]. By [16, 5.13 and 5.14], $\operatorname{cd} A=1$. So $\operatorname{cd} A=2 \operatorname{implies} \mathrm{K} \operatorname{dim} A \geq 2$. Next is a consequence of Lemma 1.7(4).

Proposition 2.1. Suppose $\operatorname{cd} A=2$. Then there are only finitely many graded primes $P \subset A$ with $\mathrm{Kdim} A / P \geq 2$. As a consequence $A$ has a finite classical Krull dimension. 
Proof. Let $I$ be the minimal injective resolution of $R$ as right $A$-module complex. Then $I^{-2}$ is the injective hull of $H^{-2} R$. Since $H^{-2} R$ is noetherian, its Goldie rank is finite and $I^{-2}$ is a finite direct sum of indecomposable injectives. By Lemma 1.7(4) and $[16,1.11]$, if $P$ is prime with $\operatorname{Kdim} A / P \geq 2$, then $E_{A / P}$ is a direct summand of $I^{-2}$. If $P \neq P^{\prime}$, then $E_{A / P} \not E_{A / P^{\prime}}$. Therefore the number of graded primes $P$ with $\mathrm{K} \operatorname{dim} A / P \geq 2$ is no more than the Goldie rank of $H^{-2} R$.

The last assertion follows from [9, I.1.9].

Lemma 2.2. Let $\operatorname{cd} A=2$ and let $M$ be a noetherian graded right $A$-module.

(1) If $\operatorname{Hom}(M, A / P) \neq 0$ for a graded prime $P$ with $\operatorname{Kdim} A / P \geq 2$, then $\operatorname{Ext}^{-2}(M, R) \neq 0$.

(2) Let $A$ be prime. If $M$ is $A$-torsion and every factor of $M$ with $\mathrm{Kdim} \geq 2$ is $A$ faithful, then $\operatorname{Ext}^{-2}(M, R)=0$.

(3) Let $A$ be prime such that, for every nonzero graded prime $P, \mathrm{Kdim} A / P \leq 1$. If $M$ is not A-torsion, then $\operatorname{Ext}^{-2}(M, R)$ is not A-torsion.

Proof. (1) Since $\operatorname{Ext}^{-2}(M, R)=\operatorname{Hom}\left(M, H^{-2} R\right)$, it suffices to show that $A / P$ as right $A$-module embeds to a finite direct sum of shifts of $H^{-2} R$. By Lemma $1.7(1,4)$, $E_{A / P}$ does not appear in $I^{0}$ and $I^{-1}$. Since $E_{A / P}$ appears in the minimal injective resolution of $R[\mathbf{1 6}, 1.11]$, it must appear in $I^{-2}$. Therefore $H^{-2} R$ contains a shift of a graded uniform right ideal of $A / P$, and hence $A / P$ embeds to a finite direct sum of shifts of $H^{-2} R$.

(2) Let $T$ be the $A$-torsion submodule of $H^{-2} R$ as right $A$-module. Then $T$ is a subbimodule and hence noetherian on both sides. Since $T_{A}$ is $A$-torsion, the right annihilator of $T$ is not zero. Suppose $\operatorname{Ext}^{-2}(M, R) \neq 0$. Then there is a nonzero map $f: M \rightarrow T$. Thus the image of $f$ has $\mathrm{Kdim} \geq 2$ and is not $A$-faithful.

(3) First we may replace $M$ by a factor of $M$ and assume $M$ is a graded uniform right ideal of $A$. Then replace $M$ by a finite direct sum of shifts of $M$ we may assume $M$ is an essential right ideal of $A$. The exact sequence

$$
0 \rightarrow M \rightarrow A \rightarrow A / M \rightarrow 0
$$

yields a long exact sequence

$$
0 \rightarrow \operatorname{Ext}^{-2}(A / M, R) \rightarrow \operatorname{Ext}^{-2}(A, R) \rightarrow \operatorname{Ext}^{-2}(M, R) \rightarrow \cdots .
$$

Since $A / M$ is $A$-torsion, $\operatorname{Ext}^{-2}(A / M, R)=0$ [Lemma 1.6(3)]. Since $\operatorname{Ext}^{-2}(A, R)=$ $H^{-2} R$ is $A$-torsionfree [Lemma $\left.1.6(3)\right], \operatorname{Ext}^{-2}(M, R)$ is not $A$-torsion.

Lemma 2.3. Suppose $\mathrm{cd} A \leq 2$ and $M$ is a noetherian graded $A$-module.

(1) Let $E^{p, q}(M)=\operatorname{Ext}^{p}\left(\operatorname{Ext}^{q}(M, R), R\right)$. Then $E^{p, q}(M)=0$ for $(p, q)=(-2,0)$, $(-1,0),(-1,-2),(0,-2)$ and $(-2,-1)$.

(2) If $\operatorname{Ext}^{-2}(M, R) \neq 0$, then $\mathrm{Kdim}_{\mathrm{Ext}^{-2}}(M, R) \geq 2$.

Proof. (1) We have a convergent spectral sequence (E1.2) and the $E_{2}$-page of (E1.2) is

$$
\begin{array}{ccc}
E^{-2,0}(M) & E^{-1,0}(M) & E^{0,0}(M) \\
E^{-2,-1}(M) & E^{-1,-1}(M) & E^{0,-1}(M) \\
E^{-2,-2}(M) & E^{-1,-2}(M) & E^{0,-2}(M)
\end{array}
$$


with other terms being zero. The boundary maps in the $E_{r}$-page have degree $(r, r-1)$. For $(p, q)=(-2,0),(-1,0),(-1,-2),(0,-2)$,

$$
E^{p, q}(M)=E_{2}^{p, q}=E_{\infty}^{p, q}=0 .
$$

At $(p, q)=(-2,-1)$, we have $E_{3}^{-2,-1}=E_{\infty}^{-2,-1}=0$. Therefore $E^{-2,-1}(M)$ is a submodule of $E^{0,0}(M)$ which is finite dimensional [Lemma 1.3(1)]. Let $N=\operatorname{Ext}^{-1}(M, R)$. Then (E1.2) for $N$ shows that $E^{0,-2}(N)=0$, which implies that $E^{-2}(N)=E^{-2,-1}(M)$ contains no nonzero $\mathfrak{m}$-torsion submodule. Therefore $E^{-2,-1}(M)$ must be zero.

(2) Let $N=\operatorname{Ext}^{-2}(M, R)$. By (1) $\operatorname{Ext}^{p}(N, R)=0$ for $p=0,-1$. If $\operatorname{Kdim} N<2$, by Lemma 1.6(2) $\mathrm{Ext}^{-2}(N, R)=0$. By $(\mathrm{E} 1.2) N=0$.

Proposition 2.4. Suppose $\operatorname{cd} A=2$. Then the following conditions are equivalent:

(1) for any chain of graded primes $P^{\prime} \subsetneq P \subset A, \mathrm{Kdim} A / P \leq 1$.

(2) $R$ is Auslander.

(3) $\mathrm{Kdim} A=2$.

(4) $\operatorname{Kdim}_{\operatorname{Ext}^{-1}}(M, R) \leq 1$ for all noetherian graded module $M$.

Proof. (1) $\Rightarrow(2)$ By $[\mathbf{1 6}, 4.18]$ we may assume $A$ is prime. By Lemma 1.3(1), for any noetherian graded $A$-module $M, \operatorname{Ext}^{0}(M, R)$ is finite dimensional and by Lemma 1.3(2) $\operatorname{Ext}^{q}(N, R)=0$ for all $N \subset \operatorname{Ext}^{0}(M, R)$ and for all $q<0$. By the definition of cd, for all $N \subset \operatorname{Ext}^{p}(M, R)$ and for all $q<-\operatorname{cd} A, \operatorname{Ext}^{q}(N, R)=0$. Hence it remains to show that $\operatorname{Ext}^{-2}(N, R)=0$ for all $N \subset \operatorname{Ext}^{-1}(M, R)$. By Lemma 1.6(3), it suffices to show that $\operatorname{Ext}^{-1}(M, R)$ is $A$-torsion, which follows from Lemmas 2.2(3) and 2.3(1).

(2) $\Rightarrow$ (3) By $[\mathbf{1 6}, 4.14], \operatorname{Kdim} A \leq \operatorname{cd} A=2$. So $\mathrm{Kdim} A=2$.

(3) $\Rightarrow$ (1) Clear, or see Lemma 3.3(2).

$(3) \Rightarrow(4)$ Again we may assume $A$ is prime. By the proof of $(1) \Rightarrow(2)$, $\operatorname{Ext}^{-1}(M, R)$ is $A$-torsion. Hence $\operatorname{Kdim}_{\operatorname{Ext}}^{-1}(M, R) \leq \mathrm{Kdim} A-1 \leq 1$.

(4) $\Rightarrow(2)$ By the prove of $(1) \Rightarrow(2)$, it remains to show that $\operatorname{Ext}^{-2}(N, R)=0$ for all $N \subset \operatorname{Ext}^{-1}(M, R)$. Since $\operatorname{Kdim}_{\operatorname{Ext}^{-1}}(M, R) \leq 1, \operatorname{Kdim} N \leq 1$ and by Lemma 1.6(2), $\operatorname{Ext}^{-2}(N, R)=0$.

REMARK 2.5. We can also prove the following, but tedious proofs are omitted.

Suppose $\operatorname{cd} A=2$. Then the following are equivalent:

(1) $\operatorname{Kdim} A=2$.

(2) $\operatorname{Kdim} M \otimes_{A} P \leq 1$ for every graded primes $P$ with $\operatorname{Kdim} A / P=2$ and for all noetherian graded $A$-module $M$ with $\operatorname{Kdim} M=1$.

(3) $\operatorname{Kdim}_{\operatorname{Ext}}^{-1}(M, R) \leq 1$ for every noetherian graded $A$-module $M$ with $\mathrm{Kdim} M=1$.

Lemma 2.6. Suppose $\operatorname{cd} A=2$. Let $M$ be a graded $A$-bimodule noetherian on both sides.

(1) If $\operatorname{Kdim} M=1$, then $\operatorname{Ext}^{-2}(M, R)=0$ and $\operatorname{Kdim}_{\operatorname{Ext}}^{-1}(M, R)=1$.

(2) If $\operatorname{Kdim} M>1$, then $\operatorname{Kdim}_{\operatorname{Ext}}^{-2}(M, R) \geq 2$ and $\operatorname{Kdim}^{\operatorname{Ext}^{-1}}(M, R) \leq 1$.

Proof. (1) By Lemma 1.6(2), $\quad \operatorname{Ext}^{-2}(M, R)=0 . \quad$ By Lemma 1.3(3), $\mathrm{Kdim}_{\operatorname{Ext}}^{-1}(M, R)>0$. So it remains to show $\operatorname{Kdim} \operatorname{Ext}^{-1}(M, R) \leq 1$. By noetherian 
induction we may assume the bimodule $M$ is right $A / P$-torsionfree for some graded ideal $P$ and $\operatorname{Kdim} A / P=1$. Hence $\operatorname{Ext}^{-1}(M . R)$ is a noetherian left $A / P$-module, which has $\mathrm{Kdim} \leq 1$.

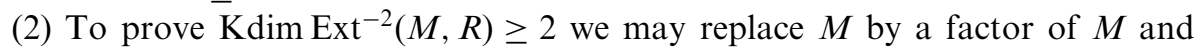
assume that every proper bimodule factor of $M$ has Kdim no more than 1. Pick a nonzero subbimodule $M^{\prime} \subset M$ such that $M^{\prime}$ is right $A / P$-torsionfree for some graded prime ideal $P$. Let $\bar{M}=M / M^{\prime}$. Then $\operatorname{Kdim} \bar{M} \leq 1$. The short exact sequence

$$
0 \rightarrow M^{\prime} \rightarrow M \rightarrow \bar{M} \rightarrow 0
$$

yields an exact sequence

$$
0 \rightarrow \operatorname{Ext}^{-2}(\bar{M}, R) \rightarrow \operatorname{Ext}^{-2}(M, R) \rightarrow \operatorname{Ext}^{-2}\left(M^{\prime}, R\right) \rightarrow \operatorname{Ext}^{-1}(\bar{M}, R) .
$$

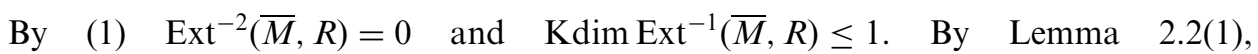
$\operatorname{Ext}^{-2}\left(M^{\prime}, R\right) \neq 0 \quad$ and $\quad$ by Lemma 2.3(2), $\quad \operatorname{Kdim}_{\operatorname{Ext}^{-2}}\left(M^{\prime}, R\right)>1$. Hence $\operatorname{Kdim} \operatorname{Ext}^{-2}(M, R) \geq 2$.

Proposition 2.7. Suppose $\operatorname{cd} A=2$.

(1) Let $M$ be a graded A-bimodule noetherian on both sides. If $M \supset M_{1} \supset M_{2} \supset \cdots$ is a descending chain of subbimodules, then $\mathrm{K} \operatorname{dim} M_{i} / M_{i+1} \leq 1$ for $i \gg 0$. As a consequence $\operatorname{Kdim}_{A^{e}} M \leq 2$.

(2) $\operatorname{Kdim}_{A^{e}} A \leq 2$.

Proof. (2) is a special case of (1).

(1) Let $N_{i}=M / M_{i}$ and let $M_{i}^{\prime}=M_{i} / M_{i+1}$. Suppose Kdim $M_{i}^{\prime}>1$ for all $i$. The exact sequence

$$
0 \rightarrow M_{i}^{\prime} \rightarrow N_{i+1} \rightarrow N_{i} \rightarrow 0
$$

yields an exact sequence

$$
0 \rightarrow \operatorname{Ext}^{-2}\left(N_{i}, R\right) \rightarrow \operatorname{Ext}^{-2}\left(N_{i+1}, R\right) \rightarrow \operatorname{Ext}^{-2}\left(M_{i}^{\prime}, R\right) \rightarrow \operatorname{Ext}^{-1}\left(N_{i}, R\right) \rightarrow \cdots .
$$

By Lemma 2.6, $\operatorname{Kdim}_{\operatorname{Ext}^{-1}}\left(N_{i}, R\right) \leq 1$ and $\operatorname{Kdim}_{\operatorname{Ext}^{-2}}\left(M_{i}^{\prime}, R\right) \geq 2$. Hence the injective map $\operatorname{Ext}^{-2}\left(N_{i}, R\right) \rightarrow \operatorname{Ext}^{-2}\left(N_{i+1}, R\right)$ is not surjective for all $i$. Thus $\left\{\operatorname{Ext}^{-2}\left(N_{i}, R\right) \mid i \geq 0\right\}$ is an ascending chain of submodules of $\operatorname{Ext}^{-2}(M, R)$, which contradicts to the fact $\operatorname{Ext}^{-2}(M, R)$ is noetherian.

Proposition 0.5 follows from Corollary 2.1 and Proposition 2.7.

REMARK 2.8. (1) We don't know whether or not $\operatorname{Kdim} A=2$ implies $\mathrm{GK} \operatorname{dim} A=2$.

(2) If $A$ is PI or FBN or more generally satisfies similar submodule condition, then $R$ is Auslander and $\mathrm{Kdim} A=\mathrm{GKdim} A=\operatorname{cd} A$ [16, 5.13 and 5.14].

\section{Graded dimension function.}

Definition 3.1. A graded dimension function is a map $\partial$ sending finitely generated right (and left) graded modules over a connected graded ring to the set of $-\infty$, all real numbers, and all ordinals $\geq \omega$ satisfying the following properties: 
(d0) $\partial 0=-\infty$, and $\partial M=0$ if and only if $M$ is finite dimensional;

(d1) If $M$ is infinite dimensional, then $\partial M \geq 1$;

(d2) $\partial M=\max \{\partial(M / N), \partial N\}$ for any $N \subset M$ (this property is called exactness);

(d3) If $x$ is a regular element of $M=A / P$ for some prime ideal $P \subset A$, then $\partial M \geq \partial(M / x M)+1$

(d4) $\partial M=\partial(M(1))$ where $M(1)$ is the degree shift of $M$.

The graded dimension function is slightly different from the dimension function in the ungraded case (see $[\mathbf{8}$, Sect. 6.8.4] or $[\mathbf{1 6}, 2.4]$ ) because we add some natural conditions (d0), (d1) and (d4) concerning graded modules. Given a finitely generated graded module $M, \mathrm{Kdim} M$ in the graded module category is equal to $\mathrm{Kdim} M$ in the ungraded module category. Using this fact one can easily check that Krull dimension is also a graded dimension function. For Gelfand-Kirillov dimension, $(\mathrm{d} 0, \mathrm{~d} 1, \mathrm{~d} 3, \mathrm{~d} 4)$ are clear and the next lemma shows that $(\mathrm{d} 2)$ is true.

Lemma 3.2. Let $A$ be a right noetherian connected ring and let $M$ be a finitely generated right graded $A$-module. Then

(1) $\mathrm{GKdim} M=\lim \log _{n}\left(\sum_{i \leq n} \operatorname{dim}_{k} M_{i}\right)$.

(2) $\mathrm{GK} \operatorname{dim} M=\max \{\mathrm{GK} \operatorname{dim} M / N, \mathrm{GK} \operatorname{dim} N\}$ for any graded submodule $N \subset M$.

(3) If $f: M(-d) \rightarrow M$ is an injective map for some integer $d>0$, then $\mathrm{GKdim} M / f(M)=\mathrm{GKdim} M-1$.

Proof. (1) This follows from the facts that $A$ is a finitely generated algebra and that $M$ is a finitely generated right $A$-module.

(2) By [8, 8.3.2(ii)], $\mathrm{GKdim} M \geq \max \{\mathrm{GK} \operatorname{dim} M / N, \mathrm{GK} \operatorname{dim} N\}$. Let $\alpha$ be any number bigger than $\max \{\mathrm{GK} \operatorname{dim} M / N, \mathrm{GK} \operatorname{dim} N\}$. Then there is a constant $C$ such that

$$
\sum_{i \leq n} \operatorname{dim}_{k}(M / N)_{i} \leq C n^{\alpha} \quad \text { and } \quad \sum_{i \leq n} \operatorname{dim}_{k} N_{i} \leq C n^{\alpha}
$$

for $n \gg 0$. Thus $\sum_{i \leq n} \operatorname{dim}_{k} M_{i} \leq 2 C n^{\alpha}$ for $n \gg 0$. By (1) $\operatorname{GKdim} M \leq \alpha$.

(3) By $[8,8.3 .5], G K \operatorname{dim} M / f(M) \leq \mathrm{GKdim} M-1$. To prove the opposite inequality let $\alpha$ be any number bigger than $\operatorname{GKdim} M / f(M)$. Let $\operatorname{dim}_{k}(M / f(M))_{i}=$ $h(i)$. Then there is a constant $C$ such that $\sum_{j \leq i} h(j) \leq C i^{\alpha}$ for all $i>0$. Let $\operatorname{dim}_{k} M_{i}=$ $g(i)$. Then

$$
g(i)=h(i)+h(i-d)+h(i-2 d)+\cdots \leq \sum_{j \leq i} h(j) \leq C i^{\alpha},
$$

whence $\sum_{j \leq i} g(j) \leq C^{\prime} i^{\alpha+1}$ for some constant $C^{\prime}$ and for $i>0$. Therefore $\mathrm{GK} \operatorname{dim} M \leq \alpha+1$.

Lemma 3.3. Let $\partial$ be a graded dimension function.

(1) If $\partial\left(A_{A}\right)<2$, then $A$ is PI of $\mathrm{Kdim} \leq 1$.

(2) If $\partial\left(A_{A}\right)<3$, then, for any chain of graded primes $P^{\prime} \subsetneq P \subset A, \operatorname{Kdim} A / P \leq 1$.

Proof. (1) We may assume $A$ is prime and not $k$. Since $A$ is noetherian, by [9, I.1.6], there is a homogeneous regular element $x \in A$ of positive degree. Then $\partial(A / x A) \leq \partial A-1<1$. Hence $\partial(A / x A)=0$ and $A / x A$ is finite dimensional. Therefore $\mathrm{GKdim} A=1$ and $A$ is PI of $\mathrm{Kdim} 1$ [Lemma 1.4]. 
(2) Since $P / P^{\prime}$ is a nonzero prime ideal of $A / P^{\prime}, \partial\left(A / P^{\prime}\right)<3$ implies $\partial(A / P)<2$. Then the statement follows from (1) .

Let $R$ be a balanced dualizing complex. The canonical dimension is defined by

$$
\operatorname{Cdim} M=-\min \left\{i \mid \operatorname{Ext}^{i}(M, R) \neq 0\right\} .
$$

Note that $\operatorname{cd} A=\operatorname{Cdim} A$. If $R$ is Auslander, then Cdim is a dimension function for ungraded modules $[\mathbf{1 6}, 2.10]$. To verify Cdim is a graded dimension function, we need to check (d0), (d1) and (d4). But (d0) and (d1) follow from Lemma 1.3(2,3) and (d4) is clear for Cdim. Therefore we have:

Proposition 3.4. If $R$ is Auslander, then Cdim is a graded dimension function.

Proof of Theorem 0.1. By Proposition 2.4, (1), (2) and (4) are equivalent.

$(2) \Rightarrow(3)$ is clear and (3) $\Rightarrow(4)$ is Lemma $3.3(2)$.

$(1) \Rightarrow(5)$ is Proposition 3.4 .

(5) $\Rightarrow$ (7) By $[16,4.14], K \operatorname{dim} M \leq \mathrm{Cdim} M$. It remains to show $\mathrm{Cdim} M \leq$ $\mathrm{K} \operatorname{dim} M$. This is clear when $\mathrm{K} \operatorname{dim} M=0$ or 2 . So we only consider the case when $\mathrm{K} \operatorname{dim} M=1$. Since both dimension functions are exact, we may use noetherian induction on $M$. So we may assume $M$ is critical faithful over $A / P$ for some graded prime $P$. There are two cases. Case 1 is when $\mathrm{Kdim} A / P=2$. Since $\mathrm{Kdim} A=2, P$ is minimal. Hence $M$ is $A / P$-torsion. Thus $\operatorname{Cdim} M \leq \operatorname{Cdim} A / P-1 \leq 1$. Case 2 is when $\mathrm{Kdim} A / P \leq 1$. Then by Lemma 3.3(1), $A / P$ is PI and of GKdim $\leq 1$. Hence $\operatorname{Cdim} A / P \leq 1$ and hence $\operatorname{Cdim} M \leq 1$.

(7) $\Rightarrow$ (6) Clear.

$(6) \Rightarrow(1)$ By the proof of Proposition 2.4, it suffices to show $\operatorname{Ext}^{-2}(N, R)=0$ for all submodules $N \subset \operatorname{Ext}^{-1}(M, R)$ and for all noetherian graded module $M$. By Lemma 2.3(1) (for $(p, q)=(-2,-1)), \operatorname{Cdim} \operatorname{Ext}^{-1}(M, R)<2$. Since Cdim is exact, $\operatorname{Cdim} N<2$, i.e., $\operatorname{Ext}^{-2}(N, R)=0$.

4. AS-Gorenstein rings of injective dimension 2. By [14, 4.14] a noetherian ASGorenstein ring $A$ of injective dimension $d$ has a balanced dualizing complex $R=A^{\sigma}[-d](-e)$ for some graded automorphism $\sigma$ of $A$ and some integer $e$ in the Definition 0.3(1).

Proposition 4.1. If $A$ is $A S$-Gorenstein of injective dimension 2 and has an artinian quotient ring (with respect to the set of the regular elements of $A$ ), then $A$ is Auslander-Gorenstein.

Note that Proposition 4.1 is $[7,5.13]$ without the hypothesis that $\mathrm{GKdim} A=2$. It also follows from Theorem 0.4 that the artinian quotient ring of $A$ is in fact self-injective.

Proof. By Theorem 0.1, it suffices to show that, for any chain of graded primes $P^{\prime} \subsetneq P \subset A, \mathrm{Kdim} A / P \leq 1$. Suppose this is not true, namely, there are graded primes $P^{\prime} \subsetneq P \subset A$ such that $\mathrm{Kdim} A / P>1$. By Lemma 2.2(1),

$$
\operatorname{Hom}(A / P, A) \cong \operatorname{Ext}^{-2}(A / P, R(e)) \cong \operatorname{Ext}^{-2}(A / P, R)(e) \neq 0
$$


because $R=A^{\sigma}[-2](-e)$. Let $Q$ be the artinian quotient ring of $A$. Then $\operatorname{Hom}(A / P, A) \neq 0$ implies that $A / P \otimes_{A} Q \neq 0$. Next we show that this contradicts to the fact $P$ is not a minimal prime. By $[\mathbf{8}, 4.1 .3(\mathrm{iv})], A / N(A) \otimes_{A} Q=Q / N(Q):=Q^{\prime}$ where $N(-)$ is the prime radical of - , and $Q / Q(N)$ is the artinian quotient ring of $A / N(A)$. Since $P$ is not a minimal prime, $P / N(A)$ is an essential (left and right) ideal of $A / N(A)$. By Goldie's theorem, $Q^{\prime} / P Q^{\prime}=0$. Hence

$$
A / P \otimes_{A} Q=Q / P Q=Q^{\prime} / P Q^{\prime}=0 .
$$

This contradicts to $A / P \otimes_{A} Q \neq 0$.

Let $\partial$ be a (graded) dimension function. A nonzero (graded) module $N$ is called $p$-pure with respect to $\partial$ if $\partial M=p$ for all nonzero noetherian (graded) submodule $M \subset N$. In most cases we will take $\partial=\operatorname{Cdim}$. Let $R$ be the balanced dualizing complex over $A$ and let $\partial$ be a graded dimension function. Following Yekutieli's definition [15, 2.3], $R$ is called residue complex over $A$ (with respect to $\partial$ ) if (i) each $A$ bimodule $R^{q}$ is graded injective on both sides and (ii) each $A$-bimodule $R^{q}$ is pure of $\partial$-dimension $-q$ on both sides. Note that in [15] this is called strong residue complex, and later in [17], this is called residue complex. By [15, 2.6], if $R$ is a residue complex over $A$ with respect to $\partial$, then $R$ is Auslander and $\partial M=\operatorname{Cdim} M$ for all noetherian graded modules $M$.

We are ready to prove Theorem 0.4 .

Proof of Theorem 0.4. First of all Theorem 0.4(1) is equivalent to Theorem 0.1 (1) for AS-Gorenstein rings. 4.1.

$(1) \Rightarrow(2)$ follows from $[\mathbf{1 6}, 6.23 .3],(2) \Rightarrow(3)$ is clear and (3) $\Rightarrow(1)$ is Proposition

$(1) \Rightarrow(4)$ is $[16,6.23 .2]$ and $(4) \Rightarrow(1)$ follows from the implication $(2) \Rightarrow(1)$ of Theorem 0.1 .

$(5) \Rightarrow(1)$ is $[15,2.6]$.

(1) $\Rightarrow(5)$ By Theorem 0.1(6) $\operatorname{Cdim} M=\operatorname{Kdim} M$ for graded noetherian modules $M$. (Note that $\operatorname{Cdim} M$ and $\mathrm{Kdim} M$ could be different for ungraded module $M$ though both dimensions are well-defined.) Hence purity with respect to Cdim is equivalent to purity with respect to Kdim .

Take the minimal injective resolution of the left $A$-module complex $R=A^{\sigma}[-2](-e)$, say

$$
\cdots 0 \rightarrow I^{-2} \rightarrow I^{-1} \rightarrow I^{0} \rightarrow 0 \cdots
$$

By Lemma 1.7(1), $I^{0}=A^{\prime}$ which is 0 -pure. Let $Q$ be the (ungraded) artinian quotient ring of $A$. Since $I^{-2}$ (as ungraded module) is a submodule of $Q$ and since $Q$ is 2pure as an ungraded module, $I^{-2}$ is 2-pure. Since $Q / A$ is $A$-torsion, so is $I^{-2} / A(-e)$. Thus $\operatorname{Cdim} N \leq 1$ for all noetherian submodule $N \subset I^{-2} / A(-e)$. Since $A$ is ASGorenstein, $I^{-2} / A(-e) \subset I^{-1}$ contains no finite dimensional submodules. Therefore $I^{-2} / A(-e)$ is 1 -pure. Since the complex (E4.2) is exact at $I^{-1}$ and since $I^{0}$ is 0 -pure, $I^{-1}$ is 1-pure. Similarly, the minimal injective resolution of the right $A$-module complex $R$ has a pure resolution. By [17, 3.8], the Cousin complex $E R$ of $R$ is a residue complex, namely, $E R$ is a balanced dualizing complex in $D_{f g}^{b}\left(\mathrm{Gr} A^{e}\right)$ which is a pure minimal injective resolution on both sides. 
$(5) \Rightarrow(6)$ By (5) $A$ is 2-pure with respect to Cdim and $\mathrm{Kdim}$.

(6) $\Rightarrow$ (1) follows from Theorem 0.1 because $\mathrm{K} \operatorname{dim} A=2$.

Remark 4.3. Part (5) is a generalization of a result of Ajitabh [1, 3.12], which proves the existence of residue complexes for AS-regular algebras of global dimension 2.

Corollary 4.4. Let $A$ be AS-Gorenstein of injective dimension 2. If $\partial$ is a graded dimension function such that $\partial \leq 2$ then

$$
\text { ว } M=\mathrm{K} \operatorname{dim} M=\mathrm{Cdim} M
$$

for all noetherian graded left and right A-modules $M$.

Proof. First of all, by Theorem 0.1, $A$ is Auslander-Gorenstein and $\mathrm{Cdim} M=\mathrm{K} \operatorname{dim} M$. So it remains to show that $\partial M=\mathrm{K} \operatorname{dim} M$.

Let $P$ be a minimal prime of $A$. By Theorem $0.4(4) \mathrm{Kdim} A / P=2$, whence $A /(P+x A)$ is not finite dimensional over $k$ for any $x$ of positive degree. Pick $\bar{x}:=x+P$ a homogeneous regular element of $A / P$ of positive degree. Then $\partial A / P \geq \partial A /(P+x A)+1 \geq 2$. Thus $\partial A=\partial(A / P)=2$ for all minimal prime $P$. Since both Kdim and $\partial$ are exact we may use noetherian induction and may assume $M$ is a critical module and faithful over $A / P$ for a graded prime $P$. If $P=\mathfrak{m}$, then $M$ is $k$ and hence both dimensions are 0 . If $M$ is infinitely dimensional and either $P$ is not minimal or $P$ is minimal and $M$ is $A / P$-torsion, then both dimensions are 1 (by (d1) and (d3)). The last possibility is when $M$ is a right ideal of $A / P$ when $P$ is minimal. In this case, $\mathrm{K} \operatorname{dim} M=\mathrm{K} \operatorname{dim} A / P$ and $\partial M=\partial A / P$. We have already shown that both dimensions on $A / P$ are equal to 2 . That completes our proof.

AcKnowledgements. This research was finished during the first author's visit to the Department of Mathematics at the University of Washington supported by a research fellowship from the China Scholarship Council and he thanks these two institutions for the hospitality and the support. The second author was supported in part by the NSF and a Sloan Research Fellowship.

\section{REFERENCES}

1. K. Ajitabh, Residue complex for regular algebras of dimension 2, J. Algebra 179 (1996), No. 1, 241-260.

2. M. Artin, J. Tate and M. Van den Bergh, Some algebras associated to automorphisms of elliptic curves, in The Grothendieck Festschrift, Vol. I, (ed. P. Cartier et al.), (Birkhäuser, 1990), 33-85.

3. M. Artin, J. Tate and M. Van den Bergh, Modules over regular algebras of dimension 3, Invent. Math. 106 (1991), No. 2, 335-388.

4. M. Artin and J. J. Zhang, Noncommutative projective schemes, Adv. Math. 109 (1994), 228-287.

5. E. K. Ekström, The Auslander condition on graded and filtered Noetherian rings. Lecture Notes in Mathematics, No. 1404, (Springer-Verlag, 1989), 220-245,

6. G. R. Krause and T. H. Lenagan, Growth of algebras and Gelfand-Kirillov dimension, Revised Edition, Graduate Studies in Mathematics, Vol. 22 (AMS, Providence, Rhode Island, 1999). 
7. T. Levasseur, Some properties of noncommutative regular rings, Glasgow Math. J. 34 (1992), 277-300.

8. J. C. McConnell and J. C . Robson, Noncommutative Noetherian rings (Wiley, 1987).

9. C. Năstăsescu and F. van Oystaeyen, Graded ring theory (North-Holland Publishing Co., 1982).

10. L. W. Small and R. B. Warfield Jr., Prime affine algebras of Gelfand-Kirillov dimension one, J. Algebra 91 (1984), No. 2, 386-389.

11. S. P. Smith and J. T. Stafford, Regularity of the four-dimensional Sklyanin algebra, Compositio Math. 83 (1992), No. 3, 259-289.

12. J. Tate and M. Van den Bergh, Homological properties of Sklyanin algebras, Invent. Math. 124 (1996), No. 1-3, 619-647.

13. M. Van den Bergh, Existence theorems for dualizing complexes over non-commutative graded and filtered rings, J. Algebra 195 (1997), No. 2, 662-679.

14. A. Yekutieli, Dualizing complexes over noncommutative graded algebras, J. Algebra 153 (1992), 41-84.

15. A. Yekutieli, The residue complex of a noncommutative graded algebra, J. Algebra 186 (1996), 522-543.

16. A. Yekutieli and J. J. Zhang, Rings with Auslander dualizing complexes, J. Algebra 213 (1999), No. 1, 1-51. print.

17. A. Yekutieli and J. J. Zhang, Residual complex over noncommutative rings, pre- 